\title{
Carbono almacenado en la biomasa aérea de plantaciones de hule (Hevea brasiliensis Müell. Arg.) de diferentes edades
}

\section{Stored carbon in the aboveground biomass of rubber (Hevea brasiliensis Müell. Arg.) plantations at different ages}

\author{
Liliana Y. López-Reyes', Marivel Domínguez-Domínguez²*, Pablo Martínez-Zurimendi3,4, Joel Zavala-Cruz², \\ Armando Gómez-Guerrero 5 y Saúl Posada-Cruz ${ }^{6}$
}

1 Colegio de Postgraduados. Campus Tabasco. Programa docente de Postgrado en Producción Agroalimentaria en el Trópico. Cárdenas, Tabasco México.

2 Colegio de Postgraduados. Campus Tabasco. Área de Ciencia Ambiental. Cárdenas, Tabasco México.

* Autor de correspondencia. mdguez@colpos.mx

\author{
3 El Colegio de la Frontera Sur, Unidad Villahermosa. \\ Villahermosa, Tabasco, México. \\ 4 Universidad de Valladolid. Instituto Universitario de \\ Gestión Forestal Sostenible. Palencia, España
}

5 Colegio de Postgraduados Campus Montecillo. Programa Forestal. Texcoco, Estado de México, México. 6 Universidad de Chiapas. Facultad de Ciencias Agrícolas. Área de protección vegetal. Huehuetan, Chiapas, México.

\section{RESUMEN}

Las plantaciones de hule (Hevea brasiliensis Müell. Arg.) contribuyen a la reducción de dióxido de carbono en la atmósfera al fijar carbono a través de la fotosíntesis y almacenarlo en cada uno de los componentes del árbol (hojas, ramas, fustes, cortezas y raíces) y por la transformación de residuos orgánicos en materia orgánica estabilizada en el suelo. El objetivo del presente estudio fue determinar el carbono almacenado en la biomasa aérea de plantaciones de hule en edades de 5, 9, 15, 25, 32 y 51 años, mediante el uso de inventario dasométrico y ecuaciones alométricas en Tabasco, México. El carbono almacenado se determinó a través de la regresión lineal y se analizó mediante comparación de medias de Tukey $(\mathrm{p}<0.05)$ con el programa Statistical Analysis System (SAS). El contenido de carbono varía en cada una de las edades; en la plantación de 51 años el carbono almacenado en el fuste fue $192.32 \mathrm{Mg}^{-1}$, en las ramas fue 64.75 $\mathrm{Mg} \mathrm{ha}^{-1}$ y el total aéreo del árbol fue $257.07 \mathrm{Mg} \mathrm{ha}^{-1}$, mientras que en la plantación de 5 años los valores fueron $16.65 \mathrm{Mg}^{-1}$, $9.63 \mathrm{Mg} \mathrm{ha}^{-1}$ y $26.28 \mathrm{Mg} \mathrm{ha}^{-1}$, respectivamente. Los resultados indican que $H$. brasiliensis puede considerarse como una fuente potencial de mitigación ante el cambio climático.

PALABRAs Clave: captura de carbono, ecuaciones alométricas, inventario forestal, plantaciones forestales, servicios ambientales.

\section{ABSTRACT}

Hevea brasiliensis Müell. Arg plantations contribute to the reduction of atmospheric carbon dioxide by fixing carbon through photosynthesis and storing it in the tree components (leaves, branches, stems, barks and roots) and by their transformation to stabilized forms of soil organic matter. The objective of this study was to determine the stored carbon in the whole aboveground biomass in 5 , 9 , 15, 25, 32 and 51-year-old plantations, through a forest inventory and the use of allometric equations in Tabasco, Mexico. The stored carbon was determined using linear regression and mean values were analyzed by the Tukey test $(\mathrm{p}<0.05)$ with the Statistical Analysis System (SAS) program. Results indicated that the carbon concentration varies with age; in 51-year-old plantation the stored carbon in stem was $192.32 \mathrm{Mg} \mathrm{ha}^{-1}$, in branches was $64.75 \mathrm{Mg} \mathrm{ha}^{-1}$ and in the whole tree was $257.07 \mathrm{Mg}^{-1}$, while 5-year plantations had values of $16.65,9.63$ y $26.28 \mathrm{Mg} \mathrm{ha}^{-1}$, respectively. The results indicated that $H$. brasiliensis plantations can be considered a potential source of mitigation of climate change.

KEYWORDs: carbon sequestration, allometric equations, forest inventory, forest plantations, environmental services. 


\section{INTRODUCCIÓN}

El carbono es un componente básico y fundamental para la vida debido a su presencia en la atmósfera, en lo vegetal, animal, en la materia orgánica no viva, en los combustibles fósiles, en las rocas y también está disuelto en los océanos (McVay y Rice, 2002). El ciclo del carbono se caracteriza por tener reservas atmosféricas muy pequeñas, pero sumamente activas y vulnerables a las perturbaciones ocasionadas por el hombre, las cuales, a su vez, modifican los patrones climáticos, de manera que afectan directamente la vida sobre la tierra. Durante la última mitad del siglo XX la concentración de dióxido de carbono $\left(\mathrm{CO}_{2}\right)$ en la atmósfera ha tenido un aumento significativo junto con la de otros gases de efecto invernadero que retienen un porcentaje de la radiación de onda larga reflejada por la superficie terrestre. Esto contribuye con casi 1/6 de las emisiones de $\mathrm{CO}_{2}$ mundial cuando los bosques han sido talados o quemados en exceso, debido a que los árboles están compuestos de carbono en un $50 \%$, y una vez talados y quemados o descompuesta la materia orgánica por microorganismos, ese carbono que almacenan regresa a la atmósfera. Por esta razón, los ecosistemas forestales son de gran importancia, ya que capturan el dióxido de carbono de la atmósfera a través de la fotosíntesis y almacenan el carbono en cada uno de los componentes del árbol (Percy, Jandl, Hall y Lavigne, 2003; Álamo, 2007).

México se ubica dentro de las 20 naciones con mayor emisión de $\mathrm{CO}_{2}$, pero al mismo tiempo tiene un alto potencial de recursos naturales como bosques, selvas y plantaciones comerciales, que son de gran importancia como almacén de carbono (Pacheco-Escalona et al., 2007). Los árboles en particular, asimilan y almacenan grandes cantidades de carbono durante toda su vida (Ordoñez, de Jong y Masera, 2001). Una alternativa para mitigar el cambio climático se lograría mediante plantaciones de corta rotación y árboles de rápido crecimiento. Al mismo tiempo, la producción de árboles en plantación reduce la extracción de madera del bosque nativo y contribuye a la conservación del mismo.
Las plantaciones forestales y agroforestales capturan carbono y lo almacenan principalmente en la biomasa dura (madera), hojas, ramas, corteza y raíces; esto depende de la composición de las especies en un bosque o plantación, del efecto de factores climáticos locales como precipitación y temperatura, de las características del suelo que determinan el crecimiento del árbol (Somarriba et al., 2013), de la edad y de la fauna acompañante. Además, la capacidad de capturar carbono atmosférico tiende a disminuir en relación al incremento de la edad del bosque o al grado de madurez del suelo, en edades tempranas o intermedias es más alta la captura de carbono (Fonseca, Rey-Benayas y Alice, 2011; Ajit et al., 2013).

México cuenta con plantaciones de Hevea brasiliensis Müell. Arg. en Veracruz, Oaxaca, Chiapas y Tabasco, con una superficie plantada a nivel nacional de 27165.98 ha de las cuales 4243.13 ha pertenecen al estado de Tabasco en los municipios de Huimanguillo, Macuspana, Jalapa y Teapa (Servicio de Información Agroalimentaria y Pesquera, 2013).

Se han realizado estudios de estimación de carbono en $H$. brasiliensis en monocultivos y sistemas agroforestales en la Amazonia de Colombia a través de modelos alométricos (Moreno, Burgos, Nieves y Buitrago, 2005; Orjuela, Andrade y Vargas, 2014); ecuaciones de aditividad para estimar componentes de biomasa de $H$. brasiliensis, en Veracruz, México (Monroy y Návar, 2004); análisis del crecimiento y producción de látex en plantaciones forestales comerciales de $H$. brasiliensis en Oaxaca, México (Rojo, Martínez y Jasso, 2011); potencial de secuestro de carbono en plantaciones de árboles en África (Kongsager, Napier y Mertz, 2013) entre otros estudios.

\section{OBJETIVo}

Determinar el carbono almacenado en la biomasa aérea de plantaciones de hule (Hevea brasiliensis) a diversas edades, mediante el uso de inventario dasométrico y ecuaciones alométricas en Tabasco, México. 


\section{MATERIALES Y MÉTODOS}

\section{Área de estudio}

Para realizar el estudio se utilizó el padrón de productores de la Secretaría de Desarrollo Agropecuario, Forestal y Pesca (Sedafop) y Sistema Producto Hule. Los criterios que se aplicaron para la selección de las plantaciones de $H$. brasiliensis, fueron conforme a las etapas preproductiva (5 años) y productiva con tres edades: 9 años (juventud), 15 años y 25 años (adulto), 32 años y 51 años (vejez); las edades se determinaron con base en la fecha de plantación registrada en el padrón y se verificaron con los productores. Al mismo tiempo, se consideró que las plantaciones pertenecieran a un mismo grupo de suelo para evitar influencia de sus características en los resultados (Tabla 1). Posteriormente, se realizó el recorrido en campo para localizar a los productores y seleccionar las plantaciones de $H$. brasiliensis de las edades mencionadas.

El estudio se realizó en seis plantaciones de $\mathrm{H}$. brasiliensis, ubicadas en tres ejidos del municipio de Huimanguillo, Tabasco, en la región tropical húmeda de México. Las plantaciones de 5 años, 9 años, 15 años y 25 años corresponden al ejido Pedregal Moctezuma 1a. Sección; ubicadas en la zona geográfica 15, en las coordenadas UTM: longitud y latitud; 433614-1953399, 433935195299, 433551-1953473, y 433942-1953473 respectivamente. La plantación de 32 años se ubica en el ejido Chicoacán con coordenadas UTM: longitud 447299 y latitud 1965282; y la plantación de 51 años pertenece al ejido Guadalupe Victoria con coordenadas; longitud 426988 y latitud 1950044 (Fig. 1). Los climas que predominan en el municipio de Huimanguillo son: Af (m) cálido húmedo con lluvias todo el año y Am (f) cálido húmedo con abundantes lluvias en verano (Instituto Nacional de Estadística y Geografía, 2010). La temperatura mínima mensual es de $23.3{ }^{\circ} \mathrm{C}$, la máxima de $32.3{ }^{\circ} \mathrm{C}$ y la precipitación anual es de $2360 \mathrm{~mm}$ (Comisión Nacional del Agua-Servicio Meteorológico Nacional, 2010).

Según Ortiz, Siebe y Cram (2005) la zona de estudio corresponde a la unidad ecogeográfica, llamada "terraza o planicies estructurales” de edad miocénica, porque con- forma un paisaje de lomeríos suaves. El suelo que predomina es del grupo Acrisol ácido, rico en materia orgánica y pobre en bases intercambiables (Palma-López, CisnerosDomínguez, Moreno-Cáliz y Rincón-Ramírez, 2007; Zavala-Cruz et al., 2014).

En la zona de estudio predomina vegetación secundaria de selva, así como plantaciones de H. brasiliensis, eucalipto (Eucalyptus grandis, Eucalyptus urophylla), teca (Tectona grandis), melina (Gmelina arborea), plantaciones agrícolas de naranja, limón, piña, y gran extensión de pastizales (Larios-Romero y Hernández, 1987; ZavalaCruz et al., 2014). La fauna es escasa sin embargo, se pueden encontrar tepezcuintle (Agouti paca) y mono sarahuato (Allonata palliata), tortugas como el guao (Staurotypus triporcatus) y el pochitoque (Kinosternon leucostomum) y especies de aves como el jabirú (Jabiru mycteria), el chejé (Melanerpes aurifrons) y el pistoqué (Pitangus sulphuratus).

\section{Muestreo de parcelas}

En el estudio se realizó un inventario forestal en seis plantaciones, en cada una se establecieron tres parcelas cuadradas de $20 \mathrm{~m} \times 20 \mathrm{~m}\left(400 \mathrm{~m}^{2}\right)$, obteniendo un área inventariada total de las seis plantaciones de $7200 \mathrm{~m}^{2}$. Cada parcela está conformada de 17 a 29 árboles, variando la densidad de los árboles en cada plantación, obteniendo un inventario de 400 árboles en el total de las plantaciones. A cada árbol se le midieron las variables: diámetro a la altura de pecho (d) con la forcípula y altura total del árbol (h) con la pistola Haga. De las seis plantaciones inventariadas, cuatro se encuentran en aprovechamiento de látex (9, 15, 25 y 32 años); la plantación de 5 años aún no está en aprovechamiento y la de 51 años ya terminó su etapa productiva. Con las variables obtenidas en el inventario se generó información de área basal, volumen total mediante la ecuación de cubicación de Gómez-González (2015), biomasa de fuste, biomasa de ramas, biomasa aérea total, carbono en fuste, carbono en ramas y carbono total del árbol, para determinar el carbono almacenado en la parte aérea de cada una de las plantaciones de las edades de $5,9,15,25,32$ y 51 años. 


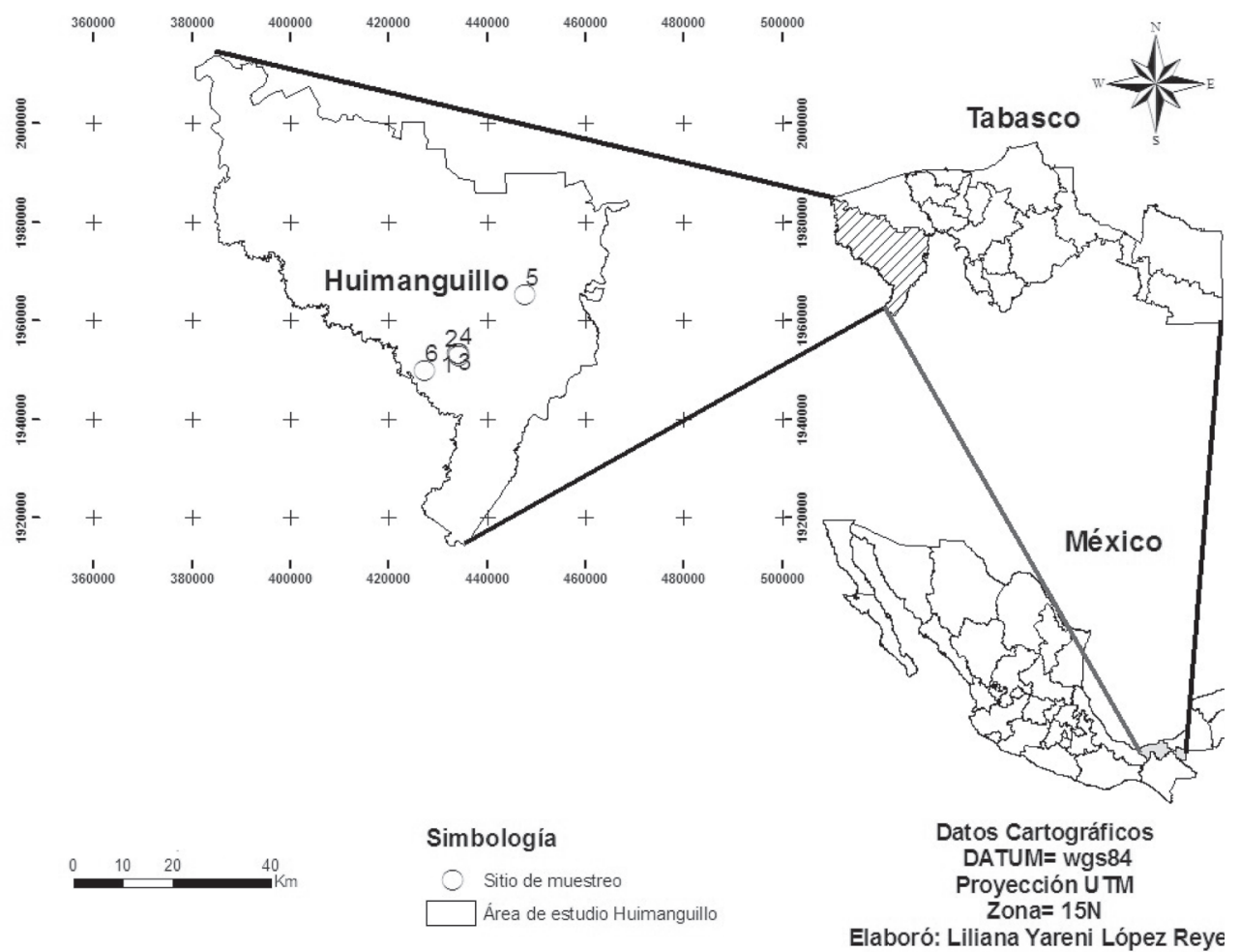

Figura 1. Ubicación geográfica de las plantaciones de H. brasiliensis en Huimanguillo, Tabasco, México.

Taвla 1. Edad de los árboles de H. brasiliensis de acuerdo con la etapa de preproductiva y productiva.

\begin{tabular}{cc}
\hline $\begin{array}{c}\text { Edad de árboles } \\
\text { (años) }\end{array}$ & Etapa preproductiva y productiva \\
\hline 5 & Crecimiento \\
9 & Juventud \\
15 & Adulto 1 \\
25 & Adulto 2 \\
32 & Vejez 1 \\
51 & Vejez 2 \\
\hline
\end{tabular}

Las mediciones que se realizaron en los árboles fueron a partir del diámetro mínimo a la altura de pecho de $8 \mathrm{~cm}$, debido a que a partir de este diámetro se obtienen algunos de los productos de Hevea brasiliensis como: muebles, molduras, mosaicos de madera, entarimado de pisos, contrachapado, tableros enlistonados, aglomerados de cemento, tablero de fibra de densidad media, estacas, polines y postes. También este diámetro se utiliza como materia prima para la industria del papel, para producir papeles gruesos y secantes o absorbentes (Zerpa, Mogollón, Gutiérrez y Aguilera, 2011).

\section{Biomasa aérea (fuste y ramas) y carbono almacenado}

Inicialmente se cuantificó la biomasa en los componentes de cada árbol que integra la plantación (hojas, ramas y fuste) (Ordoñez et al., 2001). Para cuantificar la biomasa aérea en las plantaciones de $H$. brasiliensis se utilizaron las ecuaciones generadas por Monroy y Návar (2004) para plantaciones de la misma especie en Veracruz, México, a través de regresión lineal.

$$
\begin{aligned}
& \mathrm{b}_{\text {fuste }}=37.35705+0.013422 \times \mathrm{d}^{2} \times \mathrm{h} \\
& \mathrm{b}_{\text {ramas }}=29.99596+0.004273 \times \mathrm{d}^{2} \times \mathrm{h} \\
& \mathrm{b}_{\text {total }}=67.35301+0.017695 \times \mathrm{d}^{2} \times \mathrm{h}
\end{aligned}
$$


Donde:

d (diámetro en $\mathrm{cm}$ )

$\mathrm{h}$ (altura en $\mathrm{m}$ )

$\mathrm{b}_{\text {fuste }}$ (biomasa de fuste en $\mathrm{kg}$ )

$\mathrm{b}_{\text {ramas }}$ (biomasa de ramas en $\mathrm{kg}$ )

$\mathrm{b}_{\text {total }}$ (biomasa aérea total en $\mathrm{kg}$ )

Para estimar el carbono almacenado asociado a la biomasa aérea, se multiplicó el valor de la biomasa de cada componente (fuste, ramas y total aéreo) por el factor 0.487 , valor correspondiente a las concentraciones de carbono obtenidas en la especie (Wauters, Coudert, Grallien, Jonard y Ponette, 2008; Kongsager et al., 2013).

\section{Análisis estadístico}

Los datos se sometieron a un análisis de varianza (Anova) y comparación de medias de Tukey $(\mathrm{p}<0.05)$ con el programa Statistical Analysis Sistem (SAS) para determinar la diferencia estadística en las plantaciones de diversas edades.

\section{Resultados}

\section{Historial de manejo}

El historial de manejo de las plantaciones de H. brasiliensis puede influir en el crecimiento y desarrollo de los árboles así como en la captura y almacenamiento de carbono.

Los productores establecieron el cultivo de $H$. brasiliensis con el apoyo económico federal del Fideicomiso para la Investigación, el Cultivo y la Comercialización del Hule Natural (Fidhule) para el establecimiento y manejo silvícola. Los productores asociaron cultivos agrícolas en la etapa preproductiva ( 3 años), mientras las plantaciones alcanzaron perímetros de $45 \mathrm{~cm}$ a $1.30 \mathrm{~m}$ de altura para ser aprovechado el látex. Esta asociación de cultivos agrícolas contribuye a la reducción de erosión de suelo, aporta nutrimentos en el suelo y controla malezas. Simultáneamente se realizó el manejo silvícola de las plantaciones y poda de formación para obtener un fuste limpio para ser aprovechado. Esta actividad se realizó en los primeros años debido al cierre de dosel arbóreo que se presentó a partir de los 5 años (Tabla 2).

\section{Inventario forestal}

Con respecto a la medición de las variables realizada en el inventario forestal de las plantaciones de $\mathrm{H}$. brasiliensis se presenta lo siguiente:

Diámetro (d) y altura de los árboles (h)

El análisis de varianza mostró diferencia altamente significativa $(\mathrm{P} \leq 0.0001)$ en el diámetro y altura en cada plan-

Tabla 2. Antecedentes, asociación de cultivos en callejones y densidad actual de las plantaciones de H. brasiliensis en Tabasco.

\begin{tabular}{|c|c|c|c|c|c|}
\hline $\begin{array}{c}\text { Año de } \\
\text { establecimiento }\end{array}$ & $\begin{array}{c}\text { Edad de la } \\
\text { plantación (años) }\end{array}$ & $\begin{array}{c}\text { Vegetación anterior a } \\
\text { H. brasiliensis } \\
\end{array}$ & $\begin{array}{l}\text { Densidad actual de la } \\
\text { plantación (árb.ha-1) }\end{array}$ & $\begin{array}{l}\text { Cultivo intercalado en } \\
\text { los tres primeros años }\end{array}$ & $\begin{array}{c}\text { Apoyo } \\
\text { económico }\end{array}$ \\
\hline 2009 & 5 & Pastizal & 491 & Piña (Anana sp). & \\
\hline 2005 & 9 & Pastizal & 550 & Piña (Anana sp). & \\
\hline 1999 & 15 & Acahual & 700 & Piña (Anana sp). & \\
\hline 1989 & 25 & Acahual & 633 & $\begin{array}{l}\text { Maíz (Zea mays), frijol } \\
\text { (Phaseolus vulgaris L.) y } \\
\text { yuca (Manihot sculenta) }\end{array}$ & $\begin{array}{c}\text { Planta y manejo } \\
\text { sílvicola }\end{array}$ \\
\hline 1982 & 32 & Pastizal & 558 & $\begin{array}{l}\text { Maíz (Zea mays), frijol } \\
\text { (Phaseolus vulgaris L.) }\end{array}$ & $\begin{array}{c}\text { Planta y manejo } \\
\text { silvícola }\end{array}$ \\
\hline 1963 & 51 & Acahual y Pastizal & 400 & Maíz (Zea mays) & $\begin{array}{c}\text { Planta y manejo } \\
\text { silvícola }\end{array}$ \\
\hline
\end{tabular}


tación de las edades de 5, 9, 15, 25, 32 y 51 años, con una probabilidad $(\mathrm{p}<0.05)$. La comparación de medias de Tukey mostró que el diámetro de la plantación de 15 y 25 años de edad, son similares estadísticamente entre ellas y diferentes con las otras plantaciones.

La plantación de 5 a 9 años de edad, presentó un incremento de $2 \mathrm{~cm}$ de diámetro (Tabla 3 ) sin diferencia estadística; sin embargo, entre la plantación de 9 y de 15 años de edad hubo un incremento de diámetro de $8.6 \mathrm{~cm}$, con diferencia significativa estadísticamente. Asimismo, de la plantación de 15 a 25 años de edad se presentó un incremento de $2.08 \mathrm{~cm}$, aun cuando hay una diferencia de 10 años entre las dos edades, el incremento fue relativamente pequeño y estadísticamente no presentó diferencias en el diámetro. De la misma manera, de la plantación de 25 a la de 32 años de edad se presentó un incremento de $5.58 \mathrm{~cm}$, sin diferencias significativas. No obstante, la plantación de 32 a 51 años de edad presentó un incremento de $15.12 \mathrm{~cm}$ en un lapso de 19 años, presentando diferencia altamente significativa, probablemente favorecido por la densidad actual de 400 árboles ha ${ }^{-1}$ en la plantación de 51 años y 558 árboles ha ${ }^{-1}$ en la de 32 años (Tabla 3).

La altura total de los árboles mostró diferencia significativa en las edades de 9 y 15 años con un incremento en $8 \mathrm{~m}$; la mayor altura promedio de los árboles se obtuvo en la plantación de 51 años de edad (Tabla 3).

TABla 3. Comparación de medias, diámetro, altura, área basal H. brasiliensis, en Tabasco, México.

\begin{tabular}{cccc}
\hline Edad (Años) & $\begin{array}{c}\text { Diámetro } \\
(\mathrm{cm})\end{array}$ & Altura $(\mathrm{m})$ & $\begin{array}{c}\text { Área Basal } \\
\left(\mathrm{m}^{2} . \mathrm{ha}^{-1}\right)\end{array}$ \\
\hline 5 & $14.05 \mathrm{~d}$ & $11.33 \mathrm{~b}$ & $7.88 \mathrm{c}$ \\
9 & $16.05 \mathrm{~d}$ & $13.39 \mathrm{~b}$ & $11.81 \mathrm{c}$ \\
15 & $24.65 \mathrm{c}$ & $21.41 \mathrm{a}$ & $35.12 \mathrm{~b}$ \\
25 & $26.73 \mathrm{cb}$ & $22.99 \mathrm{a}$ & $39.42 \mathrm{~b}$ \\
32 & $32.31 \mathrm{~b}$ & $23.26 \mathrm{a}$ & $49.60 \mathrm{~b}$ \\
51 & $47.43 \mathrm{a}$ & $24.22 \mathrm{a}$ & $80.41 \mathrm{a}$ \\
\hline
\end{tabular}

Medias con letras distintas son estadísticamente diferentes (Tukey, $\mathrm{p}<0.05$ ).
En la comparación de medias por Tukey en la plantación de 51 años se obtuvo la máxima área basal con valor de $80.41 \mathrm{~m}^{2} \mathrm{ha}^{-1}$, indicando diferencia altamente significativa con cada una de las plantaciones. El área basal presentó diferencias estadísticas similares al diámetro, ya que es una variable que depende directamente de él, siendo influenciada también por la edad y por el manejo silvícola. Al mismo tiempo, mostró incrementos de (3.91, 23.31, $4.3,10.18$ y 30.81$) \mathrm{m}^{2} \mathrm{ha}^{-1}$, entre las edades de plantación de $(5,9,15,25,32$ y 51$)$ años, respectivamente.

\section{Volumen de madera en los árboles}

El volumen de madera en los árboles en relación a la edad de las plantaciones presentó diferencia altamente significativa $(\mathrm{P} \leq 0.0001)$ con una probabilidad $(\mathrm{p}<0.05)$ en el análisis de varianza. El coeficiente de determinación fue de $\mathrm{R}^{2}=0.94$ (Tabla 4).

En la prueba de comparación de medias por Tukey, se obtuvo diferencia estadística significativa en la plantación de 51 años con valor promedio de $865 \mathrm{~m}^{3} \mathrm{ha}^{-1}$ de volumen maderable respecto a las demás (Fig. 2). Asimismo, se observó diferencia de $396 \mathrm{~m}^{3}$ ha $^{-1}$ entre la plantación de 32 y 51 años de edad; y diferencia de $239 \mathrm{~m}^{3} \mathrm{ha}^{-1}$ entre las plantaciones de 9 y 15 años de edad.

\section{Biomasa de fuste, ramas y total en los árboles}

La plantación de 51 años de edad presentó mayor biomasa de fuste, ramas y total aérea que las demás por el tamaño que adquirió durante su ciclo de crecimiento y desarrollo en diámetro y altura. El fuste aportó $63 \%$ y $66 \%$ de bio-

Tabla 4. Análisis de varianza de volumen de madera en plantaciones de diversas edades de $H$. brasiliensis.

\begin{tabular}{cccccc}
\hline FV & GL & SC & CM & FC & Pr>F \\
\hline Modelo & 5 & 1395001.41 & 279000.28 & 40.33 & $<.0001$ \\
Error & 12 & 83022.75 & 6918.56 & & \\
Total & 17 & 1478024.16 & & & \\
C.V. $=23.73$ & & & & & \\
\hline
\end{tabular}




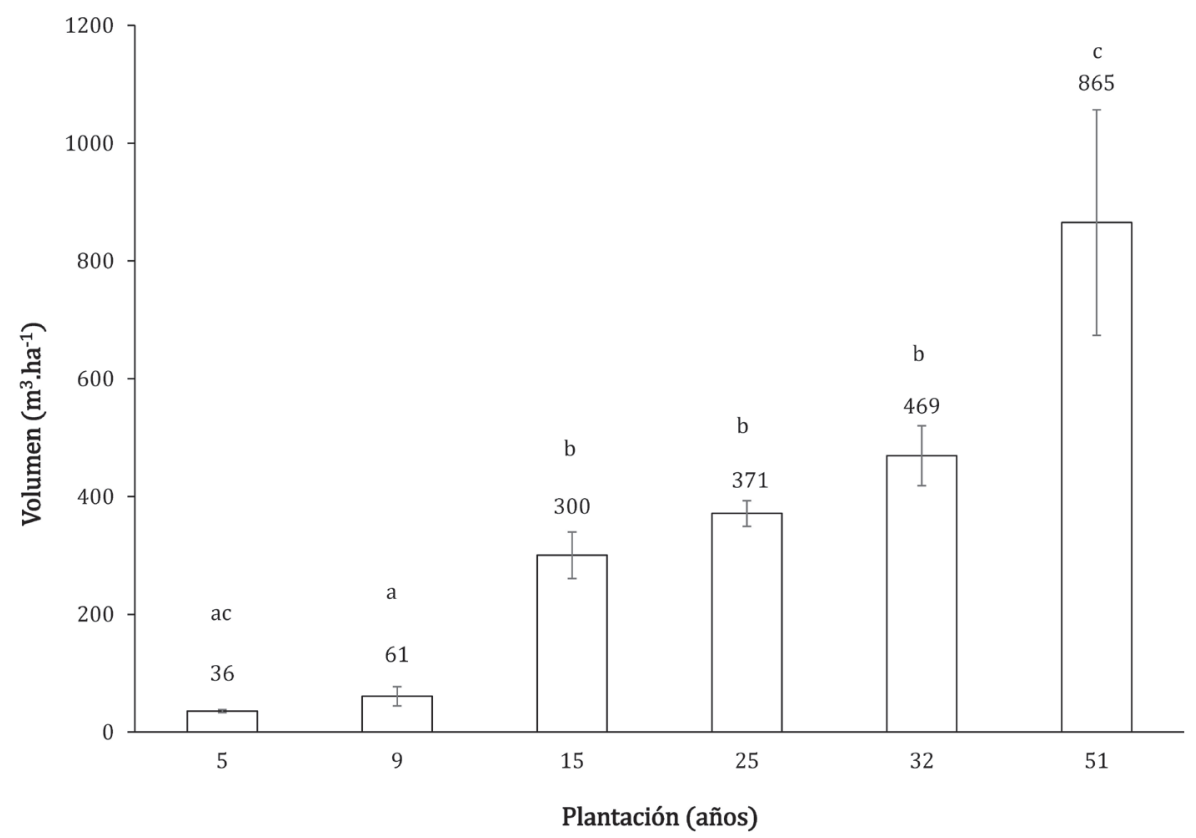

Figura 2. Comparación de medias de volumen de madera $\left(\mathrm{m}^{3} \mathrm{ha}^{-1}\right)$ en plantaciones de diversas edades de $H$. brasiliensis, en Tabasco, México.

Tabla 5. Comparación de medias por Tukey, biomasa de fustes, ramas y total aéreo para H. brasiliensis, en Tabasco, México.

\begin{tabular}{|c|c|c|c|c|c|c|}
\hline $\begin{array}{l}\text { Edad } \\
\text { (años) }\end{array}$ & $\begin{array}{l}\text { Biomasa fuste } \\
\qquad\left(\text { t.ha }^{-1}\right)\end{array}$ & $\begin{array}{c}\text { Biomasa ramas } \\
\left(\text { (t.ha-1) }^{-1}\right.\end{array}$ & $\begin{array}{l}\text { Biomasa total } \\
\text { aérea (t.ha-1) }\end{array}$ & $\begin{array}{c}\text { Carbono } \\
\text { fuste (Mg.ha-1) }\end{array}$ & $\begin{array}{l}\text { Carbono ramas } \\
\qquad\left(\mathrm{Mg}^{\left.-\mathrm{ha}^{-1}\right)}\right.\end{array}$ & $\begin{array}{l}\text { Carbono total } \\
\text { aéreo (Mg.ha-1) }\end{array}$ \\
\hline 5 & 34.18 & 19.78 & 53.96 & $16.65 c$ & $9.63 c$ & $26.28 \mathrm{c}$ \\
\hline 9 & 48.50 & 25.39 & 73.50 & $23.62 c$ & $12.37 c$ & $35.79 c$ \\
\hline 15 & 159.48 & 63.44 & 222.93 & 77.67 b & $30.89 \mathrm{~b}$ & $108.57 b$ \\
\hline 25 & 180.51 & 68.93 & 249.44 & $87.91 \mathrm{~b}$ & 33.57 b & $121.48 \mathrm{~b}$ \\
\hline 32 & 228.01 & 82.70 & 310.71 & 111.04 b & $40.27 b$ & $151.32 \mathrm{~b}$ \\
\hline 51 & 394.91 & 132.96 & 527.87 & $192.32 \mathrm{a}$ & 64.75 a & 257.07 a \\
\hline
\end{tabular}

Medias con letras distintas son estadísticamente diferentes (Tukey, $\alpha=0.05$ ).

masa en las edades de 5 y 9 años respectivamente y 73 y $75 \%$ en las edades de 32 y 51 años (Tabla 5).

\section{Estimación de carbono almacenado en las plantaciones}

En la prueba de Tukey de carbono almacenado de cada componente del árbol (fuste, ramas y total aéreo), la plan- tación de la etapa vejez de 51 años de edad mostró diferencia altamente significativa $(\mathrm{P} \leq 0.0001)$ respecto a las plantaciones de 5, 9, 15, 25 y 32 años de edad. El carbono almacenado de cada componente del árbol en la plantación de 51 años (etapa vejez) fue $192.32 \mathrm{Mg} \mathrm{ha}^{-1}$ en el fuste, $64.75 \mathrm{Mg} \mathrm{ha}^{-1}$ en las ramas y $257.07 \mathrm{Mg} \mathrm{ha}^{-1}$ total aérea. Asimismo, las plantaciones de 32 y 51 años (etapa 
vejez) mostraron diferencia estadística en el carbono almacenado de cada uno de los componentes del árbol de $81.28 \mathrm{Mg} \mathrm{ha}^{-1}$ en el fuste, $24.48 \mathrm{Mg} \mathrm{ha}^{1}$ en las ramas y 105.75 $\mathrm{Mg} \mathrm{ha}^{-1}$ en total aéreo.

Por otra parte, el carbono almacenado en fuste, ramas y total aéreo no presentó diferencia estadística entre las plantaciones de 5 y 9 años. Entre las plantaciones de 15, 25 y 32 años de edad no se presentó diferencia estadística en cada uno de los componentes del árbol (fuste, ramas y total aéreo), debido a la cercanía de edades (Tabla $5)$.

El análisis de varianza mostró diferencia altamente significativa $(\mathrm{P} \leq 0.0001)$ en el carbono almacenado en cada uno de los componentes del árbol (fuste, ramas y total de la parte aérea) y entre las edades, con una probabilidad de $(\mathrm{p}<0.05)$ (Tabla 6$)$.

\section{DISCUSIÓN}

La densidad actual de las plantaciones está influida por la asociación de cultivos agrícolas que los productores intercalaron en la etapa preproductiva, lo cual favorece a las plantaciones con menor densidad en obtener mayor desarrollo en el diámetro, aunque la disminución de árboles a partir de la densidad inicial de establecimiento también depende de los daños mecánicos y del raleo natural de estas plantaciones (Naji, Sahri, Nobuchi y Bakar, 2012). Además se realizó el manejo silvícola de podas de ramas primarias a partir de 3 o 4 años de edad para obtener un fuste libre de nudos.

El incremento en diámetro de 32 a 51 años de edad fue de $15.12 \mathrm{~cm}$, en un lapso de 19 años, presentando diferencia altamente significativa, favorecido por la densidad de 400 árboles ha ${ }^{-1}$ a los 51 años y 558 árboles ha ${ }^{-1}$ a los 32 años de edad, lo cual indica que los árboles de la plantación de 51 años se desarrollaron mejor debido al espaciamiento de la plantación. Por esta razón, los árboles acumulan mayor biomasa por la disponibilidad de agua y nutrimentos que les permite tener un mejor desarrollo (Naji, Sahri, Nobuchi y Bakar, 2011).

La biomasa de fuste representó $70.20 \%$ y la de ramas $29.83 \%$ en árboles de 5, 9, 15, 25, 32 y 51 años, similar a
Tabla 6. Análisis de varianza de almacenamiento de carbono de fuste, ramas y total de la parte aérea de H. brasiliensis.

\begin{tabular}{lccccc}
\hline FV & GL & SC & CM & FC & Pr>F \\
\hline Modelo & 5 & 65452.22 & 13090.44 & 37.05 & $<.0001$ \\
Error & 12 & 4240.16 & 353.34 & & \\
Total & 17 & 69692.38 & & & \\
C.V. $=21.57$ & & & & & \\
FV & $\mathrm{GL}$ & $\mathrm{SC}$ & $\mathrm{CM}$ & $\mathrm{FC}$ & $\mathrm{Pr}>\mathrm{F}$ \\
Modelo & 5 & 6420.27 & 1284.05 & 34.4 & $<.0001$ \\
Error & 12 & 447.92 & 37.32 & & \\
Total & 17 & 6868.19 & & & \\
C.V.= 18.64 & & & & & \\
FV & $\mathrm{GL}$ & $\mathrm{SC}$ & $\mathrm{CM}$ & $\mathrm{FC}$ & $\mathrm{Pr}>\mathrm{F}$ \\
Modelo & 5 & 112932.06 & 22586.41 & 36.44 & $<.0001$ \\
Error & 12 & 7437.87 & 619.82 & & \\
Total & 17 & 120369.94 & & & \\
C.V. $=20.76$ & & & & & \\
\hline
\end{tabular}

lo encontrado por Monroy y Návar (2004), donde obtuvieron un promedio de $72.83 \%$ y $21.4 \%$, respectivamente en árboles de 8, 14, 16, 19, 23 y 31 años. A su vez presentó diferencia estadística significativa con respecto al fuste y ramas, en las diversas edades (Gayoso y Guerra, 2005; Aguilar, Ortiz, Vílchez y Chazdon, 2011).

El carbono almacenado de cada componente del árbol en la plantación de 51 años etapa vejez fue de $192.32 \mathrm{Mg}$ $\mathrm{ha}^{-1}$ en el fuste, $64.75 \mathrm{Mg} \mathrm{ha}^{-1}$ en las ramas y $257.07 \mathrm{Mg} \mathrm{ha}^{-1}$ total aéreo, este último valor se asemeja al documentado por Kongsager et al. (2013) en árboles de 44 años de 213.6 $\mathrm{Mg} \mathrm{ha}^{-1}$, sin embargo, al comparar los datos con los de un bosque tropical subcaducifolio (árboles, arbustos, renuevos, necromasa, hojarasca y raíces), selva mediana de Quercus oleoides, y plantaciones de E. urophylla y G. arborea son inferiores, ya que el almacenamiento de carbono está en función de la composición de especies, la edad, densidad, diámetro y altura (Rodríguez-Laguna, Jiménez, Meza, Aguirre y Razo, 2008; Cámara-Cabrales, AriasMontero, Martínez-Sánchez y Castillo-Acosta, 2013). 
El valor de carbono total de la plantación de 5 años es mayor a lo que informan Durán, Duque y Suárez (2011), quienes obtuvieron un promedio de $6.6 \mathrm{Mg} \mathrm{ha}^{-1}$ de carbono almacenado en la parte aérea en plantaciones de hule de 1 a 7 años, con diámetro de $8.2 \mathrm{~cm}$ y altura de 6.2 $\mathrm{m}$ en Colombia; y en un sistema agroforestal con árboles del mismo intervalo de edad 6.9 $\mathrm{Mg} \mathrm{ha}^{-1}$. En plantaciones de $H$. brasiliensis de 1 a 7 años de edad Orjuela, Andrade y Vargas (2014) midieron 4.3 $\mathrm{Mg} \mathrm{ha}^{-1}$ de carbono almacenado total aéreo en monocultivo con densidad de 622 árboles ha ${ }^{-1}$ y $4.2 \mathrm{Mg} \mathrm{ha}^{-1}$ en un sistema agroforestal con densidad de 673 árboles ha ${ }^{-1}$; mientras que en este estudio se midieron $26.28 \mathrm{Mg} \mathrm{ha}^{-1}$ con diámetro de $14.05 \mathrm{~cm} \mathrm{y}$ altura de $11.3 \mathrm{~m}$. Los mismos autores trabajaron con plantaciones de $H$. brasiliensis de 8-20 años de edad encontrando $57.2 \mathrm{Mg} \mathrm{ha}^{-1}$ de carbono almacenado total de la parte aérea en monocultivo y $44.8 \mathrm{Mg} \mathrm{ha}^{-1}$ en sistemas agroforestales, sin embargo, al compararlo con las plantaciones de este estudio en ese intervalo de edades se determinó un valor más alto, $108.57 \mathrm{Mg} \mathrm{ha}^{-1}$ a los 15 años, debido a la mayor densidad de arbolado encontrado en Tabasco. Cabe señalar que la fijación de carbono depende de las características de cada especie, tamaño de copa, densidad en árboles por hectárea, edad e índice de sitio, que influyen fuertemente en el almacenamiento de carbono (Nájera y Hernández, 2008).

Orjuela et al. (2014) mencionan que las plantaciones mayores de 20 años de edad obtuvieron promedio de 121.5 $\mathrm{Mg} \mathrm{ha}^{-1}$ en plantación monoespecífica y $97.2 \mathrm{Mg}$ $\mathrm{ha}^{-1}$ en sistemas agroforestales, y comparando las plantaciones en el grupo mayor de 25 años de edad se obtuvo un valor muy similar con promedio de $121.48 \mathrm{Mg} \mathrm{ha}^{-1}$. El carbono almacenado en los árboles está proporcionalmente relacionado con la biomasa.

De acuerdo con los resultados de Sun et al. (2015) los árboles en etapa juvenil ( 9 años de edad) fijan más carbono. Por esta razón, los árboles en la etapa joven contribuyen a la reducción del dióxido de carbono que se encuentra en la atmósfera y lo transforman en biomasa (Casanova-Lugo, Petit-Aldana y Solorio-Sánchez, 2011). Contrariamente a lo esperado, las plantaciones de edad avanzada presentaron incremento en el carbono almacenado de más de $100 \mathrm{Mg} \mathrm{ha}^{-1}$. El cultivo de H. brasiliensis contribuye a la reducción de dióxido de carbono a través de la fotosíntesis y fija o almacena el carbono en cada uno de los componentes del árbol (fuste y ramas), obteniendo un mayor almacenamiento en el fuste (Wauters et al., 2008; Fonseca et al., 2011; Casanova-Lugo et al., 2011).

Los árboles en la etapa de vejez (32 y 51 años de edad) continúan proporcionando una serie de beneficios tanto para el ser humano como para el medio ambiente, favorecen la conservación de flora y fauna, disminución de erosión de suelo y fertilización del mismo, a través de la hojarasca. En esta etapa los productores toman la decisión de aprovechar la madera de los árboles mayores de 35 años de edad, debido a la disminución de producción de látex, ya que no es rentable seguir aprovechándolo para esa actividad y por los servicios ambientales no perciben ningún apoyo económico (Matan y Matan, 2008; Prabhakaran-Nair, 2010).

\section{CONCLUSIONES}

La plantación de 51 años de edad de $H$. brasiliensis almacena mayor carbono que las plantaciones de menor edad, esto está relacionado con la mayor biomasa. La comparación de medias manifestó que el fuste contiene más carbono que las ramas. El porcentaje de carbono almacenado en el fuste va incrementándose con la edad desde $63 \%$ a los 5 años, hasta $75 \%$ a los 51 años. Las plantaciones mantienen su capacidad de captura de carbono hasta edades superiores a las productivas del cultivo del hule. Mediante la captura y el almacenamiento de carbono estas plantaciones brindan un servicio ambiental, ya que cuentan con potencial para mitigar el $\mathrm{CO}_{2}$ de la atmósfera y conservar la flora y fauna asociadas.

\section{REFERENCIAS}

Aguilar, H., Ortiz, E., Vílchez, B. y Chazdon, R. L. (2011). Biomasa sobre el suelo y carbono orgánico en el suelo en cuatro estadios de sucesión de bosques en la Península de Osa, Costa Rica. Revista Forestal Mesoamericana Kurú, 9 (22), 22-3. Recuperado de http://tecdigital.tec.ac.cr/servicios /ojs/index.php/kuru/article/view/360. 
Ajit, Dhyani, S. K., Ramnewaj, Handa, A. K., Prasad, R., Alam, B., Rizvi, R. H., Gupta, G., Pandey, K. K., Jain, A. y Uma. (2013). Modeling analysis of potential carbon sequestration under existing agroforestry systems in three districts of Indo-gangetic plains in India. Agroforestry Systems, 87(5), 1129-1146. doi: 10.1007/s10457-013-9625-x.

Álamo, J. C. (2007). Bosques y cambio climático: la función de los bosques como sumideros de carbono y su contribución al cumplimiento del Protocolo de Kioto por parte de España. Zaragoza, España: Escuela Agraria de Cogullada

Cámara-Cabrales, L. C., Arias-Montero, C., Martínez-Sánchez, J. L. y Castillo-Acosta, O. (2013). Carbono almacenado en selva mediana de Quercus oleoides y plantaciones de Eucaliptus urophylla y Gmelina arborea en Huimanguillo, Tabasco. En F. Paz-Pellat, J. Wong-González, M. Bazan y V. Saynes (Ed.), Estado Actual del Conocimiento del Ciclo del Carbono y sus Interacciones en México (249-256). Texcoco, Mx: Programa Mexicano del Carbono.

Casanova-Lugo, F., Petit-Aldana, J. y Solorio-Sánchez, J. (2011). Los sistemas agroforestales como alternativa a la captura de carbono en el trópico mexicano. Chapingo Serie Ciencias Forestales y del Ambiente, 17(1), 133-143. doi: 10.5154/r.rchscfa.2010.08.047

Comisión Nacional del agua- Servicio Meteorológico Nacional. (2010). Información Climatológica. Recuperado de http://smn.cna.gob.mx/index.php?option=com_content \&view=article\&id=168\&tmpl=component

Durán, E. H., Duque, L. y Suárez, J. C. (2011). Estimación de carbono en sistemas agroforestales de Hevea brasiliensis en la Amazonia Colombiana. Revista Ingeniería \& Amazonia, 4(1), 19-28.

Fonseca, W., Rey-Benayas, J. M. y Alice F. E. (2011). Carbon accumulation in the biomass and soil of different aged secondary forests in the humid tropics of Costa Rica. Forest Ecology and Management 262 (8), 1400-1408. doi:10.1016/j.foreco.2011.06.036.

Gayoso, J. y Guerra, J. (2005). Contenido de carbono en la biomasa aérea de bosques nativos en Chile. Bosques, 26 (2), 33-38.
Gómez-González, J. P. (2015). Producción de madera en plantaciones de bule (Hevea brasiliensis) en Huimanguillo, Tabasco. Tesis de Maestría en Ciencias; Programa Producción Agroalimentaria en el Trópico, Colegio de Postgraduados Campus Tabasco, H. Cárdenas, Tabasco. Recuperado de: (http://www.ecosur.mx/ecoconsulta/indicadores/detalles.php?id=565d16c0a5ea9de37ca09f0b\&bd $\mathrm{i}=30$ \&name $=$ Producci $\% \mathrm{C} 3 \% \mathrm{~B} 3 \mathrm{n} \% 20 \mathrm{de} \% 20 \mathrm{madera} \% 20$ en $\% 20$ plantaciones $\% 20$ de $\% 20$ hule $\% 20$ (Hevea $\% 20$ brasiliensis) $\% 20$ en $\% 20$ Huimanguillo, $\% 20$ Tabasco

Instituto Nacional de Estadística y Geografía. (2010). Prontuario de información geográfica municipal de los Estados Unidos Mexicanos, Huimanguillo, Tabasco. Recuperado en http://www3.inegi.org.mx/sistemas/mexicocifras/ datos-geograficos/27/27008.pdf

Kongsager, R., Napier, J. y Mertz, O. (2013). The carbon sequestration potential of tree crop plantations. Mitigation and Adaptation Strategies for Global Change 18 (8), 1197-1213. doi: 10.1007/s11027-012-9417-z.

Larios-Romero, J. y Hernández, J. (1987). Zonificación fisiográfica del estado de Tabasco. Revista de Geografía Agrícola, 13 y 14, 20-32.

Matan, M. y Matan, N. (2008). Antifungal activities of anise oil, lime oil, and tangerine oil against molds on rubberwood (Hevea brasiliensis). International Biodeterioration \& Biodegradation, 62 (1), 75-78.

McVay, K. A. y Rice, C. W. (2002). El carbono orgánico del suelo y el ciclo global del carbono. Recuperado de http:// www.bookstore.ksre.ksu.edu/pubs/MF2548S.pdf.

Monroy, C. y Návar, J. J. (2004). Ecuaciones de aditividad para estimar componentes de biomasa de Hevea brasiliensis Muell. Arg., Veracruz, México. Madera y bosques, 10 (2), 29-43.

Moreno, J. A., Burgos, J. D., Nieves, H. E. y Buitrago, C. E. (2005). Modelo alométrico general para la estimación del secuestro de carbono por plantaciones de caucho Hevea brasiliensis Müell Arg. en Colombia. Colombia Forestal, 91(8), 5-21. Recuperado de http://revistas.udistrital.edu. co/ojs/index.php/colfor/article/view/3043/ 4397.

Nájera, J. A. y Hernández, E. (2008). Relaciones morfométricas de un bosque coetáneo de la región de El Salto, Durango. Ra Ximbai, 4 (1), 69-81. 
Naji, H. R., Sahri, M. H., Nobuchi, T. y Bakar, E. S. (2011).The effect of growth rate on wood density and anatomical characteristics of rubber wood (Hevea brasiliensis Müell. Arg.) in two different clonal trails. Journal of Natural Products and Plant Resources, 1 (2), 71-80.

Naji, H. R., Sahri, M. H., Nobuchi, T. y Bakar, E. S. (2012). Clonal and planting density effects on some properties of rubber wood (Hevea brasiliensis Müell. Arg.). BioResources, 7 (1), 189-202.

Ordoñez, J. A., de Jong, B. H. J. y Masera, O. (2001). Almacenamiento de carbono en un bosque de Pinus pseudostrobus en Nuevo San Juan, Michoacán. Madera y Bosques 7 (2), 27-47.

Orjuela, J. A., Andrade, H. J. y Vargas, Y. (2014). Potential of carbon storage of rubber (Hevea brasiliensis Müell. Arg.) plantations in monoculture and agroforestry systems in the Colombian Amazon. Tropical and Subtropical Agroecosystems, 17 (2), 231-240.

Ortiz, M. A., Siebe, C. y Cram S. (2005). Diferenciación ecogeográfica de Tabasco. Cap. 14:305-322. En J. Bueno, F. Álvarez y S. Santiago (Eds.) Biodiversidad del estado de Tabasco, $305-316$ p. México: Instituto de Biología, Unam-Conabio.

Pacheco-Escalona, F. C., Aldrete, A., Gómez, A., Fierros, A. M., Cetina, V. M. y Vaquera, H. (2007). Almacenamiento de carbono en la biomasa aérea de una plantación joven de Pinus greggii Engelm, Revista Fitotecnia Mexicana, 30 (3). 251-254.

Palma-López D. J., Cisneros-Domínguez, J., Moreno-Cáliz, E. y Rincón-Ramírez, J. A. (2007). Suelos de Tabasco: su uso y manejo sustentable. Villahermosa, Tabasco, México: Colegio de Postgraduados-Isprotab-Fundación Produce Tabasco.

Percy, K. E., Jandl, R., Hall, J. P. y Lavigne, M. (2003). El papel de los bosques en ciclo, la captura y el almacenamiento de carbono. Recuperado de http://iufro-archive.boku.ac.at/ iufro/taskforce/tfcs/issue1_march31_Spanisch.pdf

Prabhakaran-Nair, K. P. (2010). Rubber (Hevea brasiliensis). En K. P. Prabhakaran-Nair (Ed.), The Agronomy and Economy of Important Tree Crops of the Developing World. (p. 237-273). Londres: Elsevier.
Rodríguez-Laguna, R., Jiménez, J., Meza, J., Aguirre, O. y Razo, R. (2008). Carbono contenido en un bosque tropical subcaducifolio en la reserva de la biosfera El Cielo, Tamaulipas, México. Revista Latinoamericana de Recursos Naturales, 4 (2), 215 -222.

Rojo, G. E., Martínez, R. y Jasso, J. (2011). El cultivo del hule en México. México: Universidad Autónoma Indígena de México- Colegio de Postgraduados. Campus Montecillo Programa de Forestal. Recuperado de http://www.uaim. $\mathrm{mx} /$ Documentos/CultivoDelHule.pdf.

Servicio de Información Agroalimentaria y Pesquera. (2013). Sector presupuestal: agricultura ganadería, desarrollo rural, pesca y alimentación. Recuperado de http://www. siap.gob.mx/cierre-de-la-produccion-agricola-por-cultivo/. Consultado el 18 de abril 2015.

Somarriba, E., Cerda, R., Orozco, L., Cifuentes, M., Dávila, H., Espin, T., Mavisoya, H., Ávila, G., Alvarado, E., Poveda, V., Astorga, C., Say, E. y Deheuvels, O. (2013). Carbon stocks and cocoa yields in agroforestry systems of Central America. Agriculture, Ecosystems and Environment, 173, 46-57. doi.10.1016/j.agee.2013.04.013.

Sun, J., Guan, D., Wu, J., Jing, Y., Yuan, F., Wang, A. y Jin, C. (2015). Day and night respiration of three tree species in a temperate forest of northeastern China. iForest - Biogeosciences and Forestry, 8, 25-32. doi: 10.3832/ifor0982007.

Wauters, J. B., Coudert, S., Grallien, E., Jonard, M. y Ponette, Q. (2008). Carbon stock in rubber tree plantations in western Ghana and Mato Grosso (Brazil). Forest Ecology and Management, 255, 2347-2361. doi:10.1016/j. foreco.2007.12.038.

Zavala-Cruz, J., Salgado-García, S., Marín-Aguilar, A., PalmaLópez, D. J., Castelán-Estrada, M. y Ramos-Reyes, R. (2014). Transecto de suelos en terrazas con plantaciones de cítricos en Tabasco. Revista Ecosistemas y Recursos Agropecuarios, 1 (2), 213-137.

Zerpa, J., Mogollón, G., Gutiérrez, I. y Aguilera, A. (2007). Potencial papelero de clones de Hevea brasiliensis procedentes del Estado Amazonas, Venezuela. Revista Forestal Venezolana, 51 (1), 69-76. 
Manuscrito recibido el 25 de mayo de 2015.

Aceptado el 19 de octubre de 2016.

Este documento se debe citar como:

López-Reyes, L. Y., Domínguez-Domínguez, M., Martínez-Zurimendi, P., Zavala-Cruz, J., Gómez-Guerrero, A. y Posada-Cruz, S. (2016). Carbono almacenado en la biomasa aérea de plantaciones de hule (Hevea brasiliensis Müell. Arg.) de diferentes edades. Madera y Bosques, 22 (3), 49-60. 